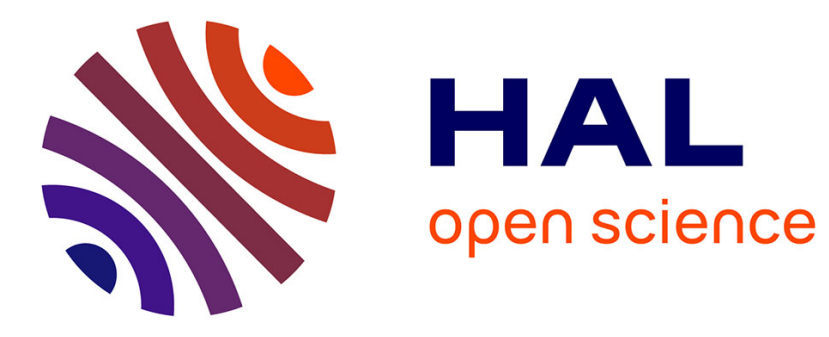

\title{
Equilibrium analysis in financial markets with countably many securities
}

\author{
Charalambos D. Aliprantis, Monique Florenzano, Victor-Filipe \\ Martins-Da-Rocha, Rabee Tourky
}

\section{- To cite this version:}

Charalambos D. Aliprantis, Monique Florenzano, Victor-Filipe Martins-Da-Rocha, Rabee Tourky. Equilibrium analysis in financial markets with countably many securities. Journal of Mathematical Economics, 2004, 40 (6), pp.683-699. 10.1016/j.jmateco.2003.06.003 . halshs-00086810

\section{HAL Id: halshs-00086810 \\ https://shs.hal.science/halshs-00086810}

Submitted on 19 Jul 2006

HAL is a multi-disciplinary open access archive for the deposit and dissemination of scientific research documents, whether they are published or not. The documents may come from teaching and research institutions in France or abroad, or from public or private research centers.
L'archive ouverte pluridisciplinaire HAL, est destinée au dépôt et à la diffusion de documents scientifiques de niveau recherche, publiés ou non, émanant des établissements d'enseignement et de recherche français ou étrangers, des laboratoires publics ou privés. 


\title{
EQUILIBRIUM ANALYSIS IN FINANCIAL MARKETS WITH COUNTABLY MANY SECURITIES
}

\author{
C. D. ALIPRANTIS ${ }^{1}$, M. FLORENZANO 2 , V. F. MARTINS DA ROCHA ${ }^{3}$, AND R. TOURKY ${ }^{4}$
}

${ }^{1}$ Department of Economics, Krannert School of Management, Purdue University, W. Lafayette, IN 47907-1310, USA; aliprantis@mgmt.purdue.edu

2 CNRS-CERMSEM, Université Paris 1, 106-112 boulevard de l'Hôpital, 75647 Paris Cedex 13, FRANCE; monique.florenzano@univ-paris1.fr

3 CERMSEM, Université Paris 1, 106-112 boulevard de l'Hôpital, 75647 Paris Cedex 13, FRANCE; martins@univ-paris1.fr

${ }^{4}$ Department of Economics and Commerce, University of Melbourne, Parkville 3052, Melbourne, AUSTRALIA; rtourky@unimelb.edu.au

\begin{abstract}
An $F$-cone is a pointed and generating convex cone of a real vector space that is the union of a countable family of finite dimensional polyhedral convex cones such that each of which is an extremal subset of the subsequent one. In this paper, we study securities markets with countably many securities and arbitrary finite portfolio holdings. Moreover, we assume that each investor is constrained to have a non-negative end-of-period wealth. If, under the portfolio dominance order, the positive cone of the portfolio space is an $F$-cone, then Edgeworth allocations and nontrivial quasi-equilibria exist. This result extends the case where, as in Aliprantis-BrownPolyrakis-Werner (1998a), the positive cone is a Yudin cone.
\end{abstract}

KEYwORDS: Security markets; Edgeworth equilibrium; nontrivial quasi-equilibrium; inductive limit topology; $F$-cone; Riesz-Kantorovich functional

JEL Classification: D41, D51, D52, G11, G22

\section{INTRODUCTION}

We consider a finance model with $m$ investors trading securities and having identical expectations on the security payoffs. Let $E$ be a portfolio (vector) space. Given an ordered vector payoff space $X$, for example some $L_{p}(\Omega, \Sigma, P)$ for $1 \leq p \leq \infty$ and an underlying probability space $(\Omega, \Sigma, P)$, let a linear operator $R: E \rightarrow X$ define for each portfolio $z$ the payoff $R(z)$ an investor expects to receive when holding the portfolio $z$. If we suppose that $R$ is one-to-one, it is natural to order the portfolio space $E$ by the portfolio dominance ordering:

$$
z \geq_{R} z^{\prime} \text { whenever } R(z) \geq R\left(z^{\prime}\right) .
$$

Let us now assume that investors are constrained to have non-negative end-of-period wealth or, equivalently, that their portfolio set is equal to the cone $K=\left\{z \in E: \quad z \geq_{R} 0\right\}$ of positive payoff portfolios. If each investor has a strict preference over $K$ described by a correspondence $P_{i}: K \rightarrow K$ and an initial endowment of securities $\omega_{i} \in K$, then a financial market is given by:

$$
\mathcal{E}=\left(E, K,\left(P_{i}, \omega_{i}\right)_{i=1}^{m}\right) .
$$

Date: February 10, 2003. 
An equilibrium concept for this economy requires the definition of a security price vector space $E^{\prime}$, in duality with $E$. Relative to this duality, an equilibrium is as usual a pair $(p, x)$ consisting of a nonzero price $p \in E^{\prime}$ and a market clearing allocation $x=\left(x_{i}\right)_{i=1}^{m} \in K^{m}$ of investors' optimal portfolios subject to the given security prices. Our paper is a contribution to the equilibrium existence problem in this type of financial models.

The existence of equilibrium in these models was first studied by Brown-Werner (1995), and then by Aliprantis-Brown-Polyrakis-Werner (1998a), who introduced the notion of portfolio dominance ordering. As in Brown-Werner (1995), Aliprantis-Brown-Polyrakis-Werner (1998a) assume that there are countably infinitely many securities, but in Aliprantis-Brown-Polyrakis-Werner (1998a), each investor is restricted to portfolios with non-zero holdings of only finitely many securities. The portfolio space of the model is thus the vector space $\Phi$ of all eventually zero real sequences. Assuming that the cone $K$ of positive payoff portfolios is a Yudin cone, Aliprantis-Brown-PolyrakisWerner (1998a) prove that the portfolio space $\Phi$, equipped with the inductive limit topology ${ }^{1}$ relative to the family of all finite dimensional subspaces of $\Phi$, is a topological vector lattice. As the portfolio trading sets of investors coincide with the positive cone $K$ of the portfolio space, in the spirit of Mas-Colell (1986), the existence of a quasi-equilibrium with a price in $\mathbb{R}^{\mathbb{N}}$, the topological dual of $\Phi$, is mainly based on assumptions of uniform properness on $K$ of the preferences of the investors. Our purpose in this paper is to show that, in the same setting and under comparable properness assumptions, the key assumption that the cone $K$ of positive payoffs portfolios is a Yudin cone can be seriously weakened.

We assume that there is a finite number or a countable infinity of securities defined by their payoffs, and that the portfolio space $E$ is either some Euclidean space $\mathbb{R}^{J}$ or, as in Aliprantis-BrownPolyrakis-Werner (1998a), $\Phi$, both ordered by the portfolio dominance ordering and equipped with the inductive limit topology. As in Aliprantis-Brown-Polyrakis-Werner (1998a), we assume that the agents have the positive cone as their portfolio trading sets and make properness assumptions on their preferences, but we replace the lattice ordering hypothesis by the strictly weaker assumption that the positive cone is an $F$-cone. By this assumption, on which we will comment later, we mean that, under the portfolio dominance ordering, the positive cone of the portfolio space is a convex, pointed and generating cone $K=\bigcup_{n=1}^{\infty} K_{n}$, which is the union of a countable family of finite dimensional polyhedral convex cones $K_{n}$ such that each $K_{n}$ is an extremal subset (or a face) of $K_{n+1}$. Under these assumptions we shall establish that equilibrium exists. Moreover, the countably many extremal vectors of the positive cone have the same economic interpretation as the vectors of the Yudin basis assumed in Aliprantis-Brown-Polyrakis-Werner (1998a). The corresponding portfolios can be thought of as mutual funds that investors trade under the no short sales restriction.

The main tool of this extension is a result of Aliprantis-Florenzano-Tourky (2002) [Theorem 5.1] stating a sufficient condition for decentralizing Edgeworth allocations of a proper exchange economy of which the commodity space is not a vector lattice. This condition, expressed in terms of properness of the Riesz-Kantorovich functional associated to a list of continuous linear functionals on $E$, should be understood as a condition (satisfied in our model) of compatibility between the topology and the order structure of the portfolio space. The existence of Edgeworth allocations is obtained under classical continuity assumptions on the preferences of the agents. Their decentralization as nontrivial quasi-equilibria requires properness assumptions that we shall

\footnotetext{
${ }^{1}$ The definition of a Yudin cone and the properties of this topology will be discussed in Section 2 .
} 
make precise later. The double assumption on the topology of the portfolio space and its order structure guarantees that we can apply the result in Aliprantis-Florenzano-Tourky (2002).

The paper is organized as follows: In Section 2, we review the definition and properties of the inductive limit topology $\xi$ on $E$ generated by the family of its finite dimensional vector subspaces. We also define $F$-cones and establish some properties of $\xi$ when $E$ is ordered by an $F$-cone. Edgeworth and nontrivial quasi-equilibria of our financial economy are studied in Section 3. Section 4 is devoted to examples. The properness of the Riesz-Kantorovich functional is proved in the last section. This section and an appendix are the only technical parts of the paper.

\section{InduCtive Limit TOPOLOGY AND $F$-CONES}

2.1. The inductive limit topology. Let $E$ be a real vector space and let $\mathcal{F}$ be the family (directed by inclusion) of all finite dimensional vector subspaces of $E$. The inductive limit topology $\xi$ on $E$ generated by $\mathcal{F}$ is the finest locally convex topology on $E$ for which, for each $F \in \mathcal{F}$, the natural embedding $i_{F}: F \hookrightarrow E$ is $\left(\tau_{F}, \xi\right)$-continuous, where $\tau_{F}$ is the unique Hausdorff linear topology on $F$.

The fact that, as a finite dimensional space, each $F \in \mathcal{F}$ admits a unique Hausdorff linear topology $\tau_{F}$ has several remarkable consequences that are easy to derive: ${ }^{2}$

- If $\left(F_{\gamma}\right)_{\gamma \in \Gamma}$ is a family of finite dimensional vector subspaces such that each $F \in \mathcal{F}$ is contained in some $F_{\gamma}$, then $\xi$ is also the finest locally convex topology on $E$ for which, for each $\gamma \in \Gamma$, the natural embedding $i_{\gamma}: F_{\gamma} \hookrightarrow E$ is $\left(\tau_{F_{\gamma}}, \xi\right)$-continuous.

- The topology $\xi$ is also the finest locally convex topology on $E$. Consequently, $\xi$ is Hausdorff and the topological and algebraic duals $E^{\prime}$ and $E^{*}$ coincide.

- If $\left(e_{i}\right)_{i \in I}$ is a Hamel basis of $E$, then $E$ is the direct sum of the one dimensional vector spaces $\mathbb{R} e_{i}$ and $\xi$ is the finest locally convex topology on $E$ for which each natural embedding $\mathbb{R} e_{i} \hookrightarrow E$ is $\left(\tau_{\mathbb{R} e_{i}}, \xi\right)$-continuous.

- For each $\varepsilon=\left(\varepsilon_{i}\right)_{i \in I} \in(0, \infty)^{I}$, let

$$
V_{\varepsilon}:=\left\{x \in E:\left|x_{i}\right|<\varepsilon_{i} \text { for each } i \in I\right\} .
$$

The family of all subsets $V_{\varepsilon}$ is a base of neighborhoods at 0 for the topology $\xi$.

The following result is also classical; see Bourbaki (1966) [Chap. II, §4, Exercise 8]. We give its proof for the sake of completeness.

Proposition 2.1. Assume that $E$ is a real vector space with a countable Hamel basis. A subset $A$ of $E$ is $\xi$-closed if and only if for each finite dimensional vector subspace $F$ of $E$, the set $A \cap F$ is closed for the unique Hausdorff linear topology on F.

In particular, if $\mathcal{F}$ is the family of all finite dimensional subspaces of $E$, then

$$
\xi=\{V \subset E: V \cap F \text { is open in } F \text { for each } F \in \mathcal{F}\} \text {. }
$$

Proof. Let $A$ be a $\xi$-closed subset of $E$. For each finite dimensional subspace $F$ of $E$, the subset $i_{F}^{-1}(A)=A \cap F$ is closed in $F$.

Conversely, let $A$ be a subset of $E$ such that for each finite dimensional subspace $F$ of $E$, the subset $A \cap F$ is closed. If $\operatorname{dim} E<\infty$, then there is nothing to prove. Otherwise, let $\left(e_{n}\right)_{n \in \mathbb{N}}$ be a countable basis of $E$ and let $x \in E \backslash A$. We claim that there exists $\varepsilon=\left(\varepsilon_{i}\right)_{i \in \mathbb{N}} \in(0, \infty)^{\mathbb{N}}$ such that

\footnotetext{
${ }^{2}$ For details about inductive limit topologies see the monographs Bourbaki (1966), Kelley-Namioka (1963), and Schaefer (1971).
} 
$x+V_{\varepsilon} \subset E \backslash A$. For each $n \in \mathbb{N}$, we let $E_{n}$ be the vector space spanned by the vectors $\left\{e_{1}, \ldots, e_{n}\right\}$, and for each finite family $\left\{B_{1}, \ldots, B_{n}\right\}$ of subsets of $\mathbb{R}$, we let

$$
\prod_{i=1}^{n} B_{i}:=\left\{x \in E_{n}: x=\sum_{i=1}^{n} \alpha_{i} e_{i} \text { with } \alpha_{i} \in B_{i} \text { for each } i=1, \ldots, n\right\} .
$$

Without any loss of generality we can suppose that $x \in E_{1}$. Then for each $n \geq 1$ we have

$$
x \in E_{n} \backslash\left(A \cap E_{n}\right) .
$$

As $A \cap E_{n}$ is closed in $E_{n}$, it follows that there exists $\left(\varepsilon_{1}, \ldots, \varepsilon_{n}\right) \in(0, \infty)^{n}$ such that

$$
x+\prod_{i=1}^{n}\left(-\varepsilon_{i}, \varepsilon_{i}\right) \subset E_{n} \backslash\left(A \cap E_{n}\right) .
$$

Let $\alpha>1$. We claim that there exists $\varepsilon_{n+1}>0$ such that

$$
x+\prod_{i=1}^{n+1}\left(-\frac{\varepsilon_{i}}{\alpha}, \frac{\varepsilon_{i}}{\alpha}\right) \subset E_{n+1} \backslash\left(A \cap E_{n+1}\right) .
$$

To see this, suppose that the claim is not true. Then, we can construct a sequence $\left(v_{k}\right)_{k \in \mathbb{N}}$ of $E_{n+1}$ such that for each $k \in \mathbb{N}$,

$$
v_{k} \in \prod_{i=1}^{n}\left(-\frac{\varepsilon_{i}}{\alpha}, \frac{\varepsilon_{i}}{\alpha}\right) \times\left(-\frac{1}{k}, \frac{1}{k}\right) \quad \text { and } \quad x+v_{k} \in A \cap E_{n+1} .
$$

The sequence $\left(v_{k}\right)_{k \in \mathbb{N}}$ lies in a compact subset of $E_{n+1}$. Passing to a subsequence if necessary, we can suppose that $\left(v_{k}\right)_{k \in \mathbb{N}}$ converges to some $v \in E_{n+1}$. Note that

It follows that

$$
v \in \prod_{i=1}^{n}\left[-\frac{\varepsilon_{i}}{\alpha}, \frac{\varepsilon_{i}}{\alpha}\right] \times\{0\} .
$$

$$
x+v \in A \cap\left(x+\prod_{i=1}^{n}\left[-\frac{\varepsilon_{i}}{\alpha}, \frac{\varepsilon_{i}}{\alpha}\right]\right) \subset A \cap\left[x+\prod_{i=1}^{n}\left(-\varepsilon_{i}, \varepsilon_{i}\right)\right] .
$$

This contradicts (2.1) and the validity of (2.2) has been established.

In order to apply the previous claim, we consider a sequence $\left(\alpha_{n}\right)_{n \in \mathbb{N}} \in(1, \infty)^{\mathbb{N}}$ such that $^{3}$

$$
\lim _{N \rightarrow \infty} \prod_{n=1}^{N} \alpha_{n}=\prod_{n=1}^{\infty} \alpha_{n}<\infty
$$

Applying inductively $(2.2)$, we can construct a sequence $\left(\varepsilon_{n}\right)_{n \in \mathbb{N}} \in(0, \infty)^{\mathbb{N}}$ such that for each $n \in \mathbb{N}$ we have

$$
x+\prod_{i=1}^{n}\left(-\frac{\varepsilon_{i}}{\prod_{i=1}^{n} \alpha_{i}}, \frac{\varepsilon_{i}}{\prod_{i=1}^{n} \alpha_{i}}\right) \subset E_{n} \backslash\left(A \cap E_{n}\right) .
$$

Now consider $\beta \in \mathbb{R}$ such that $\beta>\prod_{n=1}^{\infty} \alpha_{n}$ and let $V$ be the following $\xi$-neighborhood of 0 :

$$
V:=\left\{x \in E: \quad x=\sum_{i \in \mathbb{N}} \alpha_{i} e_{i}, \text { where }\left|\alpha_{i}\right|<\frac{\varepsilon_{i}}{\beta} \text { for each } i \in \mathbb{N}\right\} .
$$

\footnotetext{
${ }^{3}$ For instance, let $\alpha_{n}=\exp \left(\frac{1}{n^{2}}\right)$.
} 
We assert that $x+V \subset E \backslash A$. Indeed, suppose that $(x+V) \cap A \neq \varnothing$. Then there exists some $n \in \mathbb{N}$ such that $(x+V) \cap A \cap E_{n} \neq \varnothing$. But

$$
(x+V) \cap E_{n} \subset x+\prod_{i=1}^{n}\left(-\frac{\varepsilon_{i}}{\beta}, \frac{\varepsilon_{i}}{\beta}\right) \subset x+\prod_{i=1}^{n}\left(-\frac{\varepsilon_{i}}{\prod_{i=1}^{n} \alpha_{i}}, \frac{\varepsilon_{i}}{\prod_{i=1}^{n} \alpha_{i}}\right) .
$$

This contradicts (2.3) and the proof is finished.

An immediate consequence of the preceding result is the following.

Corollary 2.1. Let $E$ be a real vector space with a countable Hamel basis. A subset $A$ of $E$ is $\xi$-closed if and only if there exists an increasing sequence $\left(F_{n}\right)_{n \in \mathbb{N}}$ of finite dimensional subspaces of $E$ such that:

1. each finite dimensional subspace $F$ of $E$ is contained in some $F_{n}$,

2. for every $n$ the set $A \cap F_{n}$ is closed in $F_{n}$.

Proof. Assume first that $A$ is $\xi$-closed. Pick an increasing sequence $\left(F_{n}\right)_{n \in \mathbb{N}}$ of finite dimensional vector subspaces such that $\bigcup_{n=1}^{\infty} F_{n}=F$. The latter shows that (1) is true. Since $\xi$ induces the Euclidean topology on each $F_{n}$, it follows that (2) also is true.

Conversely, assume that a sequence $\left(F_{n}\right)_{n \in \mathbb{N}}$ satisfies (1) and (2). In order to apply Proposition 2.1, we shall prove that for each finite dimensional vector subspace $F$ of $E$, the set $A \cap F$ is closed in $F$. Let $F \in \mathcal{F}$. Then there exists some $n$ such that $F \subset F_{n}$. Note that

$$
A \cap F=F \cap\left(A \cap F_{n}\right) .
$$

Since $A \cap F_{n}$ is closed in $F_{n}$, the subset $A \cap F$ is closed in $F$.

2.2. F-cones. For a precise definition of cones, convex cones, finite dimensional polyhedral convex cones, we refer the reader to Rockafellar (1970). We start with the definition of an $F$-cone.

Definition 2.2. Let $E$ be a real vector space. $A$ (convex) cone $K$ of $E$ is called a faced cone (or simply an $F$-cone) if $K$ is pointed (i.e., $K \cap(-K)=\{0\}$ ), generating (i.e., $K-K=E$ ) and $K=\bigcup_{n=1}^{\infty} K_{n}$, the union of a countable family of finite dimensional polyhedral convex cones $\left(K_{n}\right)_{n \geq 1}$ such that each $K_{n}$ is an extremal subset ${ }^{4}$ of $K_{n+1}$.

Remark 2.3. Note that if $K$ is an $F$-cone of $E$, then $E$ has a countable Hamel basis. Moreover, observe that an $F$-cone $K$ is generated by a countable family of vectors which is the union of the extremal directions of each $K_{n}$. Countably generated Yudin cones ${ }^{5}$ are particular cases of $F$-cones, but an $F$-cone is not necessarily a Yudin cone. For example, if $E$ is finite dimensional, an $F$-cone is necessarily a (pointed and generating) polyhedral convex cone. It is easy to check that an $F$-cone is a Yudin cone if and only if the number of its extremal directions is equal to the dimension of $E$. Assume now that $E$ is a vector space with a countably infinite Hamel basis $\left(e_{n}\right)_{n \in \mathbb{N}}$. Let $K$ be the convex cone generated by the family $\left\{e_{3}+e_{1}, e_{3}+e_{2}, e_{3}-e_{1}, e_{3}-e_{2}\right\} \cup\left\{e_{n}\right\}_{n \geq 4}$. It is easily verified that $K$ is an $F$-cone but not a Yudin cone.

\footnotetext{
${ }^{4} \mathrm{~A}$ convex subset $A$ of a convex set $C$ is an extremal subset (or a face) of $C$ if for each line segment $[y, z]$ of $C$ satisfying $(y, z) \cap A \neq \varnothing$ then $y$ and $z$ belong to $A$. If $K$ is a convex cone, then a convex subset $A$ of the cone is an extremal subset of $K$ if and only if $x=y+z$ with $x \in A$ and $y, z \in K$ imply $y, z \in A$. It follows that an extremal subset of a cone is itself a convex cone.

${ }^{5} \mathrm{~A}$ convex cone $C$ that is generated by a family $\left(e_{i}\right)_{i \in I}$ of vectors in a vector space is called a Yudin cone if each $x \in C$ has a unique representation of the form $x=\sum_{i \in I} \alpha_{i} e_{i}$, where $\alpha_{i} \geq 0$ and $\alpha_{i}=0$ for all but finitely many $i \in I$. It should be clear that the family $\left(e_{i}\right)_{i \in I}$ is linearly independent. Yudin cones were introduced and studied in Aliprantis-Brown-Polyrakis-Werner (1998b).
} 
Proposition 2.2. Every F-cone is $\xi$-closed.

Proof. Let $K$ be an $F$-cone of a vector space $E$. Since $K$ is generating, for each finite dimensional subspace $F$ of $E$ we have $F \subset K_{n}-K_{n}$ for some $n$. Moreover, it is easy to see that each $K_{n}$ is an extremal subset of $K_{n^{\prime}}$ for every $n^{\prime}>n$. Now we shall prove that for each $n \in \mathbb{N}$ we have $K \cap\left(K_{n}-K_{n}\right)=K_{n}$. Indeed, let $x \in K \cap\left(K_{n}-K_{n}\right)$. Then there exist $y, z \in K_{n}$ and $n^{\prime}>n$ such that $x \in K_{n^{\prime}}$ and $x=y-z$. It follows that $y=x+z$. Since $K_{n}$ is an extremal subset of $K_{n^{\prime}}$, we see that $x \in K_{n}$ and $K \cap\left(K_{n}-K_{n}\right) \subset K_{n}$. The reverse inclusion is obvious. Now recalling that a finite dimensional polyhedral convex cone is closed, an application of Corollary 2.1 completes the proof.

For the rest of our discussion in the paper we shall assume that $E$ is ordered by the $F$-cone $K$.

Proposition 2.3. The order intervals of $E$ lie in finite dimensional subspaces and thus are $\xi$ compact.

Proof. Let $a \in K$ satisfy $a \neq 0$. We only have to prove that the order interval $[0, a]=K \cap(a-K)$ is $\xi$-compact. Let $n$ be such that $a \in K_{n}$. For every $x \in[0, a]$, let $n^{\prime}$ be such that $x$ and $a-x$ lie in $K_{n^{\prime}}$. If $n^{\prime} \leq n$, then $x \in K_{n}$. If $n^{\prime}>n$, then from $a=x+(a-x)$ and the fact that $K$ is an $F$-cone, we deduce that $x \in K_{n}$. Thus $[0, a] \subset K_{n}$, and hence $[0, a]$ is included in the finite dimensional space $K_{n}-K_{n}$ (ordered by the polyhedral convex cone $\left.K \cap\left(K_{n}-K_{n}\right)=K_{n}\right)$. It follows that $[0, a]$ is a compact subset of $K \cap\left(K_{n}-K_{n}\right)=K_{n}$ and, in view of the definition of $\xi$, a $\xi$-compact subset of $E$.

Remark 2.4. Let $E$ be a vector space with a countable Hamel basis $\left(e_{n}\right)_{n \in \mathbb{N}}$. Let $K$ be the cone generated by the family $\left\{e_{1}-e_{n}, e_{n}\right\}_{n \geq 2}$. Since the order interval $\left[0, e_{1}\right]$ contains the family $\left\{e_{n}\right\}_{n \geq 1}$, it is not $\xi$-compact. In view of Proposition 2.3, the cone $K$ is not an $F$-cone.

\section{EquilibRium in SECURITIES MARKETS}

Let us now return to the model of our financial economy

$$
\mathcal{E}=\left(E, \xi, K,\left(P_{i}, \omega_{i}\right)_{i=1}^{m}\right),
$$

where the portfolio space $E$ is either a finite dimensional Euclidean vector space $\mathbb{R}^{J}$ or the vector space $\Phi$ of all eventually zero real sequences. In both cases, $E$ is equipped with the inductive limit topology ${ }^{6} \xi$, while the positive cone $K$ of the portfolio dominance ordering is assumed to be an $F$-cone. A Hamel basis of $E$ consists of the vectors $(0, \ldots, 0,1,0, \ldots)$ corresponding to each one of the countably many securities defining the model. According to the finite or countably infinite dimension of $E$, the algebraic and topological dual of $E$ is $\mathbb{R}^{J}$ or $\mathbb{R}^{\mathbb{N}}$ of which each element can be thought of as a list of prices for each security.

We first observe that it follows from Propositions 2.2 and 2.3 that $(E, \xi, K)$ is an ordered linear vector space equipped with a Hausdorff locally convex topology satisfying the properties:

A1: The positive cone $K$ is generating and $\xi$-closed.

A2: The order intervals of $E$ are $\xi$-bounded.

These properties are required for the economic model in Aliprantis-Florenzano-Tourky (2002).

Let $\omega=\sum_{i=1}^{m} \omega_{i}$ be the total initial endowment of securities, i.e., let $\omega$ be the market portfolio. A portfolio allocation is an $m$-tuple $x=\left(x_{i}\right)_{i=1}^{m} \in K^{m}$ such that $\sum_{i=1}^{m} x_{i}=\omega$.

\footnotetext{
${ }^{6}$ Obviously, on $\mathbb{R}^{J}$ the topology $\xi$ coincides with the Euclidean topology of $\mathbb{R}^{J}$.
} 
Definition 3.1. A pair $(x, p)$ consisting of an allocation $x$ and a non-zero linear functional $p$ is said to be:

1. A quasi-equilibrium, if $p\left(x_{i}\right)=p\left(\omega_{i}\right)$ for each $i$ and $y_{i} \in P_{i}\left(x_{i}\right)$ implies $p\left(y_{i}\right) \geq p\left(x_{i}\right)$.

2. An equilibrium, if it is a quasi-equilibrium and $y_{i} \in P_{i}\left(x_{i}\right)$ implies $p\left(y_{i}\right)>p\left(x_{i}\right)$.

Definition 3.2. A quasi-equilibrium $(x, p)$ is said to be non-trivial if for some $i$ we have

$$
\inf \left\{p\left(z_{i}\right): z_{i} \in K\right\}<p\left(\omega_{i}\right) .
$$

In this paper, we will be interested only in non-trivial quasi-equilibria. ${ }^{7}$ We now introduce the usual optimality properties of portfolio allocations.

Definition 3.3. A portfolio allocation $x$ is said to be:

1. Individually rational, if for each $i$ we have $\omega_{i} \notin P_{i}\left(x_{i}\right)$.

2. Weakly Pareto optimal, if there is no portfolio allocation $y$ satisfying $y_{i} \in P\left(x_{i}\right)$ for each $i$.

3. A core allocation, if it cannot be blocked by any coalition in the sense that there is no coalition $S$ and some $y \in K^{S}$ such that:

(a) $\sum_{i \in S} y_{i}=\sum_{i \in S} \omega_{i}$, and

(b) $y_{i} \in P_{i}\left(x_{i}\right)$ for all $i \in S$.

4. An Edgeworth equilibrium, if for every integer $r \geq 1$ the $r$-fold replica of $x$ belongs to the core of the r-fold replica of the economy $\mathcal{E}^{8}$

As well-known, an equilibrium allocation of $\mathcal{E}$ is an Edgeworth equilibrium (and hence a core, weakly Pareto optimal and individually rational allocation).

Recall that in our financial economy $\mathcal{E}$ each investor $i$ has the cone $K$ of positive payoff portfolios as her portfolio set and an initial endowment of securities $\omega_{i} \in K$. We posit on $\mathcal{E}$ the following assumptions.

C1: For each $i$ and for every $x_{i} \in K$ :

(a) $P_{i}\left(x_{i}\right)$ is convex and $x_{i} \notin P_{i}\left(x_{i}\right)$.

(b) $\left(P_{i}\right)^{-1}\left(x_{i}\right):=\left\{y_{i} \in K: x_{i} \in P_{i}\left(y_{i}\right)\right\}$ is $\xi$-open in $K$.

C2: The total initial endowment $\omega$ of securities is such that $\omega>_{R} 0$ (i.e., $\omega \in K$ and $\left.\omega \neq 0\right)$.

In view of Proposition 2.3 and the previous assumptions, it follows from Florenzano (1990) [Proposition 3] that Edgeworth equilibrium exists for $\mathcal{E}$.

We now introduce the additional assumption on $\mathcal{E}$ which will allow to decentralize with prices in $E^{\prime}$ (the topological dual of $E$ ) any Edgeworth equilibrium.

C3: For each $i$ and every weakly Pareto optimal allocation $x=\left(x_{i}\right)_{i=1}^{m}$, we have $x_{i} \in \operatorname{cl} P_{i}\left(x_{i}\right)$ and:

(a) $P_{i}\left(x_{i}\right)$ is $\xi$-open in $K$ or $P_{i}\left(x_{i}\right)=\left\{y_{i} \in K: u_{i}\left(y_{i}\right)>u_{i}\left(x_{i}\right)\right\}$ for some concave utility function $u_{i}: K \rightarrow \mathbb{R}$.

\footnotetext{
${ }^{7}$ If $(x, p)$ is some trivial quasi-equilibrium, then for every allocation $y$, the pair $(y, p)$ is also a quasi-equilibrium. If the quasi-equilibrium $(x, p)$ is non-trivial, then it is well-known that (under some additional continuity condition on preferences or concavity for utility functions and some irreducibility assumption on the economy) ( $x, p)$ is actually an equilibrium.

${ }^{8}$ The ideas in this definition go back to Debreu-Scarf (1963). An important reference is also Aubin (1979). Edgeworth equilibria were first introduced and studied in Aliprantis-Brown-Burkinshaw (1987).
} 
(b) There is a convex subset $\widehat{P}_{i}\left(x_{i}\right)$ of E such that the vector $x_{i}+\omega$ is a $\xi$-interior point of $\widehat{P}_{i}\left(x_{i}\right)$ and $\widehat{P}_{i}\left(x_{i}\right) \cap K=P_{i}\left(x_{i}\right)$.

Assumption C3(b) states that for each $i$ the preference correspondence $P_{i}$ is $\omega$-proper at every component of a weakly Pareto optimal portfolio allocation. This properness assumption was introduced by Tourky (1998) who proved that it is strictly weaker that Mas-Colell's $\omega$-uniform properness assumed by Aliprantis-Brown-Polyrakis-Werner (1998a) for preferences defined on $K$ by utility functions. The local non-satiation property assumed in C3 is implied by their assumption that the market portfolio $\omega$ is desirable.

Let $x=\left(x_{i}\right)_{i=1}^{m}$ be an Edgeworth equilibrium of $\mathcal{E}$. In view of Assumptions C1(a), C2, and C3, it follows from Aliprantis-Florenzano-Tourky (2002) [Theorem 5.1] that there exists some $p \in E^{\prime}$ such that $(x, p)$ is a non-trivial quasi-equilibrium, provided the following condition is satisfied:

B: If $f=\left(f_{1}, f_{2}, \ldots, f_{m}\right)$ is a list of $\xi$-continuous linear functionals such that $f_{i}(\omega)>0$ for each $i$, and $\mathcal{R}_{f}(\omega)=\sum_{i=1}^{m} f_{i}\left(x_{i}\right)$ for some $x=\left(x_{i}\right)_{i=1}^{m} \in K^{m}$ such that $\sum_{i=1}^{m} x_{i}=\omega,{ }^{9}$ then the Riesz-Kantorovich functional ${ }^{10} \mathcal{R}_{f}$ is $\omega$-proper at $\omega$.

We shall see in Section 5 that property $\mathbf{B}$ is true in our model. Using this, we can establish the following result which extends in several respects the main theorem (Theorem 6.1) in AliprantisBrown-Polyrakis-Werner (1998a).

Theorem 3.4. Assume that $\Phi$ is equipped with the inductive limit topology $\xi$ and that the cone of positive payoff portfolios $K$ is an $F$-cone. Under the assumptions $\boldsymbol{C 1}, \boldsymbol{C 2}$, and $\boldsymbol{C} 3$ on

$$
\mathcal{E}=\left(\Phi, \xi, K,\left(P_{i}, \omega_{i}\right)_{i=1}^{m}\right)
$$

there exists a non-trivial portfolio quasi-equilibrium.

When $E$ is finite dimensional, the preceding result specializes to the following.

Theorem 3.5. Assume that $J$ is a finite set of securities, and that the cone of positive payoff portfolios $K$ is an $F$-cone. Under the assumptions $\mathbf{C 1}, \mathbf{C 2}$, and $\mathbf{C 3}$ on

$$
\mathcal{E}=\left(\mathbb{R}^{J}, \xi, K,\left(P_{i}, \omega_{i}\right)_{i=1}^{m}\right),
$$

there exists a non-trivial portfolio quasi-equilibrium.

\section{EXAMPLES}

4.1. Finitely many securities. In the following examples, the family of available securities is finite and non-redundant, i.e., their payoffs are linearly independent.

Example 4.1. We start with a finite set $J$ of securities and a finite set $S$ of states of the world $(S \geq J)$, that is, with a finite dimensional payoff space $\mathbb{R}^{S}$ endowed with its canonical order. We also are given a one-to-one linear payoff operator $R: \mathbb{R}^{J} \rightarrow \mathbb{R}^{S}$. In this case, the cone $K$ of positive payoff portfolios is simply

We have the following properties.

$$
K=\left\{z \in \mathbb{R}^{J}: R z \geq 0\right\} .
$$

- Since the securities are non-redundant, $K$ is a polyhedral pointed convex cone.

\footnotetext{
${ }^{9}$ It follows from the definition of $\mathcal{R}_{f}$ and the $\xi$-compactness of the order intervals of $\Phi$ that the condition $\mathcal{R}_{f}(\omega)=\sum_{i=1}^{m} f_{i}\left(x_{i}\right)$ for some $x=\left(x_{i}\right)_{i=1}^{m} \in K^{m}$ such that $\sum_{i=1}^{m} x_{i}=\omega$ is satisfied for any list $f=\left(f_{1}, f_{2}, \ldots, f_{m}\right)$ of $\xi$-continuous linear functionals.

${ }^{10}$ The precise definition of $\mathcal{R}_{f}$ will be given in the following section.
} 
- If markets are complete, i.e., if $J=S$, then $K$ is generating - and actually $K$ is a Yudin cone.

- If $J<S$ (that is, if markets are incomplete), then $K$ is not necessarily generating. In order to know if the positive cone of a given financial model $K$ is an $F$-cone, one has to check, by computing the extremal rays, whether int $K \neq \varnothing$.

When the cone $K$ is generating, it is an $F$-cone which may have more extremal directions than the number of elements in $J$ (and thus it may not be a Yudin cone). It follows from Theorem 3.5 that under the assumptions $\mathbf{C 1}, \mathbf{C 2}$, and $\mathbf{C 3}$, the financial model $\mathcal{E}=\left(R^{J}, \xi, K,\left(P_{i}, \omega_{i}\right)_{i=1}^{m}\right)$ has a non-trivial portfolio quasi-equilibrium.

Assume now that the portfolio space is finite dimensional but that the payoff space is not finite dimensional.

Example 4.2 (Aliprantis-Monteiro-Tourky). Let the payoff space be $X=\mathbb{R}^{[0,2 \pi]}$ and the portfolio space $E$ be the three-dimensional space generated by the following three securities (defined by their payoff):

$$
R_{1}(s)=\cos s, \quad R_{2}(s)=\sin s, \quad \text { and } \quad R_{0}(s)=1, s \in[0,2 \pi] .
$$

It follows that the portfolio dominance order is defined by the "ice cream" cone

$$
K=\left\{\left(z_{0}, z_{1}, z_{2}\right) \in \mathbb{R}^{3}: z_{0} \geq \sqrt{z_{1}^{2}+z_{2}^{2}}\right\} .
$$

The cone $K$ is closed, generating and pointed but has infinitely many extremal vectors, and thus it is not an $F$-cone of $E$.

On this financial structure, Aliprantis-Monteiro-Tourky (2002) provide an example of a two investor economy satisfying the assumptions C1, C2, and C3, that has weak Pareto optimal allocations and Edgeworth equilibria but no non-trivial quasi-equilibrium.

4.2. Infinitely many securities. In the following examples, the payoff space $X$ is $\ell_{\infty}$ endowed with its natural order. The countably many available securities are defined by their linearly independent payoffs. Denote by $\left(e_{n}\right)_{n \geq 0}$ the canonical Hamel base of $\Phi$, i.e.

$$
e_{0}=(1,0, \ldots), \quad e_{1}=(0,1,0, \ldots), \ldots .
$$

Note that $\left(e_{n}\right)_{n \geq 0}$ is a family of linearly independent vectors of $\ell_{\infty}$.

Example 4.3. Let the family of available securities $\left(x_{n}\right)_{n \geq 0}$ be defined by

$$
x_{0}=(1,1,1,1,0, \ldots), x_{1}=(0,1,0,1,0, \ldots), x_{2}=(0,0,1,1,0, \ldots), x_{3}=(0,0,0,0,1,0 \ldots)
$$

and $x_{n}=e_{n+1}$ for each $n \geq 3$. Then for each $z \in \Phi$, we have $R(z) \geq 0$ if and only if

$$
\left\{\begin{array}{cl}
z_{0} & \geq 0 \\
z_{0}+z_{1} & \geq 0 \\
z_{0}+z_{2} & \geq 0 \\
z_{0}+z_{1}+z_{2} & \geq 0
\end{array} \text { and } \quad z_{n} \geq 0 \quad \text { for all } n \geq 3 .\right.
$$

The cone $K$ of positive payoff portfolios is generated by the family $\left\{e_{n}\right\}_{n \geq 3} \cup\left\{e_{0}-e_{1}, e_{0}-e_{2}, e_{1}, e_{2}\right\}$. This cone $K$ is not a Yudin cone but it is an $F$-cone. 
Example 4.4. Let the family of available securities $\left(x_{n}\right)_{n \geq 0}$ be defined by

$$
x_{0}=(1,1,1, \ldots) \text { and } \quad x_{n}=e_{n} \text { for all } n \geq 1 .
$$

Then for each $z \in \Phi$, we have $R(z) \geq 0$ if and only if $z_{0}+z_{n} \geq 0$ for each $n \in \mathbb{N}$. The cone $K$ of positive payoff portfolios is generated by the family $\left\{e_{n}\right\}_{n \geq 1} \bigcup\left\{e_{0}-\sum_{i=1}^{n} e_{i}\right\}_{n>1}$. Since the order interval $\left[0, e_{0}\right]$ contains the sequence $\left\{e_{n}\right\}_{n \geq 1}$, it is not $\xi$-compact. In view of Proposition 2.3, the cone $K$ is not an $F$-cone. It is now easy to construct economies $\mathcal{E}=\left(\Phi, \xi, K,\left(P_{i}, \omega_{i}\right)_{i=1}^{m}\right)$ satisfying C1, C2, and C3 whose positive cone coincide with $K$, and for which there exists neither weakly Pareto optimal allocation nor non-trivial quasi-equilibrium.

Example 4.5. Consider the cone $K$ of the portfolio space $\Phi$ defined by

$$
z_{0} \geq \sqrt{z_{1}^{2}+z_{2}^{2}} \text { and } z_{n} \geq 0 \text { for all } n \geq 0 .
$$

The cone $K$ is convex, pointed, generating and $\xi$-closed. Moreover order intervals are $\xi$-compact. Note however that the cone $K$ is not an $F$-cone. Following the example given by AliprantisMonteiro-Tourky (2002), one can construct an economy $\mathcal{E}$ satisfying C1, C2, and C3 that has weak Pareto optimal allocations, Edgeworth equilibria but no non-trivial quasi-equilibrium.

\section{Properness of the Riesz-Kantorovich functional}

Let $E$ be a real vector space and let $K$ be an $F$-cone of $E$. Following Aliprantis-TourkyYannelis $(2001)$, for any finite list $f=\left(f_{1}, \ldots, f_{m}\right)$ of continuous linear functionals on $(E, \xi)$ the Riesz-Kantorovich functional $\mathcal{R}_{f}$ is defined on $K$ by

$$
\mathcal{R}_{f}(x)=\sup \left\{\sum_{i=1}^{m} f_{i}\left(x_{i}\right): x_{i} \in K \text { for each } i \text { and } \sum_{i=1}^{m} x_{i}=x\right\} .
$$

If for each $\omega \in K$ we let

$$
P(\omega)=\left\{\omega^{\prime} \in K: \mathcal{R}_{f}\left(\omega^{\prime}\right)>\mathcal{R}_{f}(\omega)\right\},
$$

then the Riesz-Kantorovich functional $\mathcal{R}_{f}$ is said to be $\omega$-proper at $\omega$ if there exists a convex subset $\widehat{P}(\omega)$ of $E$ such that $2 \omega$ is a $\xi$-interior point of $\widehat{P}(\omega)$ and $\widehat{P}(\omega) \cap K=P(\omega)$.

We shall establish in this section that for $\omega>0$ (i.e., $\omega \in K$ and $\omega \neq 0$ ) and any finite list $f=\left(f_{1}, \ldots, f_{m}\right)$ of continuous linear functionals on $(E, \xi)$ such that $f_{i}(\omega)>0$ for each $i$, the Riesz-Kantorovich functional $\mathcal{R}_{f}$ is $\omega$-proper at $\omega$.

5.1. The finite dimensional case. Suppose in this subsection that $E$ is finite dimensional. Then the inductive limit topology $\xi$ coincides with the unique Hausdorff linear topology on $E$. Moreover, the $F$-cone $K$ is a pointed and generating polyhedral convex cone.

Proposition 5.1. For $\omega>0$ and any finite list $f=\left(f_{1}, \ldots, f_{m}\right)$ of linear functionals on $E$ such that $f_{i}(\omega)>0$ for each $i$, the Riesz-Kantorovich functional $\mathcal{R}_{f}$ is $\omega$-proper at $\omega$.

Proof. The functional $\mathcal{R}_{f}$ is continuous on $K$; for this conclusion we need Theorems 10.2 and 20.5 in Rockafellar (1970). The set $R(\omega):=\left\{\omega^{\prime} \in K: \mathcal{R}_{f}\left(\omega^{\prime}\right) \geq \mathcal{R}_{f}(\omega)\right\}$ is a polyhedral convex set; for this conclusion see Theorem 19.3 and Corollary 19.3.4 in Rockafellar (1970). So, we can express $R(\omega)$ as the set of solutions to a certain system

$$
R(\omega)=\left\{x \in E: \quad a_{i}(x) \geq \alpha_{i} \text { for all } i \in I\right\},
$$


where the finite family $\left(a_{i}, \alpha_{i}\right)_{i \in I} \in\left(E^{*}\right)^{I} \times \mathbb{R}^{I}$ is minimal. In view of the positive homogeneity of $\mathcal{R}_{f}$, one can assume $\alpha_{i} \geq 0$ for each $i \in I$. Let $I^{\prime}:=\left\{i \in I: \alpha_{i}>0\right\}$. Since $\mathcal{R}_{f}(\omega)>0$, it follows that $0 \notin R(\omega)$ and hence the set $I^{\prime}$ is nonempty. Let us denote by $I(\omega)$ the level set

$$
I(\omega):=\left\{\omega^{\prime} \in K: \mathcal{R}_{f}\left(\omega^{\prime}\right)=\mathcal{R}_{f}(\omega)\right\},
$$

and then consider the set

$$
J(\omega):=\left\{\omega^{\prime} \in R(\omega): \text { there exists } i \in I^{\prime} \text { such that } a_{i}(x)=\alpha_{i}\right\} .
$$

The proof will be completed by a series of steps.

Step 1: $I(\omega)=J(\omega)$.

Assume first $z \in I(\omega)$ and $z \notin J(\omega)$. So, for each $i \in I^{\prime}$ we have $a_{i}(z)>\alpha_{i}$. This implies that there is some $0<\lambda<1$ such that $a_{i}(\lambda z)>\alpha_{i}$ for all $i \in I^{\prime}$. Now if $i \notin I^{\prime}$, then $\alpha_{i}=0$ and so $a_{i}(\lambda z) \geq 0$, and therefore $\lambda z \in R(\omega)$. However, $z \in I(\omega)$ implies

$$
\mathcal{R}_{f}(\omega)=\mathcal{R}_{f}(z)>\lambda \mathcal{R}_{f}(z)=\mathcal{R}_{f}(\lambda z) \geq \mathcal{R}_{f}(\omega),
$$

which is a contradiction. Thus, $I(\omega) \subset J(\omega)$.

For the reverse inclusion, assume $z \in J(\omega)$ and $z \notin I(\omega)$. It follows that $\mathcal{R}_{f}(z)>\mathcal{R}_{f}(\omega)$. Therefore, if $\lambda=\mathcal{R}_{f}(\omega) / \mathcal{R}_{f}(z)$, then $0<\lambda<1$ and $\mathcal{R}_{f}(\lambda z)=\mathcal{R}_{f}(\omega)$. Hence $\lambda z \in I(\omega) \subset R(\omega)$ and thus $a_{i}(\lambda z) \geq \alpha_{i}$ for all $i \in I$. Now notice that since $z \in J(\omega)$, there exists some $i$ such that $a_{i}(z)=\alpha_{i}>0$. In particular, the vector $\lambda z \in R(\omega)$ satisfies $a_{i}(\lambda z)=\lambda a_{i}(z)<\alpha_{i}$, contrary to $\lambda z \in R(\omega)$. Hence, $J(\omega) \subset I(\omega)$ is also true and the validity of Step 1 has been established.

Clearly, Step 1 implies that $P(\omega)=R(\omega) \cap\left\{z \in E: a_{i}(z)>\alpha_{i}\right.$ for all $\left.i \in I^{\prime}\right\}$. We now define

$$
\widehat{P}(\omega)=\left\{z \in E: a_{i}(z)>\alpha_{i} \text { for all } i \in I^{\prime}\right\} .
$$

It follows from this definition that $\widehat{P}(\omega)$ is a nonempty convex open set and that $P(\omega) \subset \widehat{P}(\omega) \cap K$.

Step 2: $2 \omega \in \widehat{P}(\omega)$.

Indeed, from $\omega \in R(\omega)$, we get $a_{i}(\omega) \geq \alpha_{i}>0$ for each $i \in I^{\prime}$, and so $a_{i}(2 \omega)=2 a_{i}(\omega)>\alpha_{i}$.

Step 3: For each $i \notin I^{\prime}$ there exists some $u \in R(\omega)$ such that $a_{i}(u)=0$. Consequently, for any such $i \notin I^{\prime}$ and for each $z \in K$ we have $a_{i}(z) \geq 0$.

To establish this claim, recall first that if for some $i \in I$ one has $a_{i}(z)>\alpha_{i}$ for each $z \in R(\omega)$, then the inequality $a_{i}(z) \geq \alpha_{i}$ can be deleted from the expression of $R(\omega)$ given by $(\star)$. Since the family $\left(a_{i}, \alpha_{i}\right)_{i \in I}$ is supposed to be minimal, this proves the first assertion of Step 3.

For the second assertion of Step 3, assume that there exist $i \notin I^{\prime}$ and $z \in K$ with $a_{i}(z)<0$. Let $u \in R(\omega)$ be such that $a_{i}(u)=0$. Since $\mathcal{R}_{f}(u) \geq \mathcal{R}_{f}(\omega)>0$, it follows from the continuity of $\mathcal{R}_{f}$ at $u$, that there exists some $z^{\prime} \in K$ satisfying $a_{i}\left(z^{\prime}\right)<0$ and $\mathcal{R}_{f}\left(z^{\prime}\right)>0$. Using the positive homogeneity of $\mathcal{R}_{f}$, we can find some $\lambda \geq 1$ such that $\mathcal{R}_{f}\left(\lambda z^{\prime}\right) \geq \mathcal{R}_{f}(\omega)$. Hence $a_{i}\left(\lambda z^{\prime}\right) \geq 0$, which implies that $a_{i}\left(z^{\prime}\right) \geq 0$, contrary to $a_{i}\left(z^{\prime}\right)<0$.

Step 4: $\widehat{P}(\omega) \cap K \subset P(\omega)$.

To see this, let $z \in \widehat{P}(\omega) \cap K$. In view of the definition of $\widehat{P}(\omega)$, we have only to prove that $z \in R(\omega)$, i.e., that $z$ satisfies all the inequalities defining $R(\omega)$ in $(\star)$. For $i \in I^{\prime}$ this follows from $z \in \widehat{P}(\omega)$. For $i \notin I^{\prime}$ this follows from $z \in K$ and Step 3 .

We have thus established that the set $\widehat{P}(\omega)$ is an open convex set with $2 \omega \in \widehat{P}(\omega)$ and such that $\widehat{P}(\omega) \cap K=P(\omega)$. That is, we have proved that $\mathcal{R}_{f}$ is $\omega$-proper at $\omega$. 
5.2. The general case. In this subsection, the space $E$ is not supposed to be finite dimensional. According to Definition 2.2, the cone $K$ is pointed, generating and $K=\bigcup_{n=1}^{\infty} K_{n}$ is the union of a countable family of finite dimensional polyhedral convex cones $\left(K_{n}\right)_{n \geq 1}$ such that each $K_{n}$ is an extremal subset (or a face) of $K_{n+1}$. As noticed in Remark 2.3, observe that $E$ has a countable Hamel basis.

Proposition 5.2. For $\omega>0$ and any finite list $f=\left(f_{1}, \ldots, f_{m}\right)$ of continuous linear functionals on $(E, \xi)$ such that $f_{i}(\omega)>0$ for each $i$, the Riesz-Kantorovich functional $\mathcal{R}_{f}$ is $\omega$-proper at $\omega$.

Proof. Let $\omega>0$ and let $f=\left(f_{1}, \ldots, f_{m}\right)$ be a finite list of continuous linear functionals on $(E, \xi)$ such that $f_{i}(\omega)>0$ for each $i$. Without any loss of generality, we can suppose that $\omega \in K_{1}$. For each $n$ let $f^{n}$ be the finite list $\left(f_{1}^{n}, \ldots, f_{m}^{n}\right)$, where $f_{i}^{n} \in\left(E_{n}\right)^{*}$ is the restriction of $f_{i}$ to the subspace $E_{n}=K_{n}-K_{n}$. Since each $K_{n}$ is a face of $K$, we first have that $\mathcal{R}_{f^{n}}$ coincides with the restriction to $K_{n}$ of $\mathcal{R}_{f}$. That is,

$$
\mathcal{R}_{f^{n}}(x)=\mathcal{R}_{f}(x) \text { for all } x \in K_{n} .
$$

In particular, if we let $P_{n}(\omega):=\left\{\omega^{\prime} \in K_{n}: \mathcal{R}_{f^{n}}\left(\omega^{\prime}\right)>\mathcal{R}_{f^{n}}(\omega)\right\}$, then $P_{n}(\omega)=P(\omega) \cap K_{n}$. Now if we let $R_{n}(\omega):=\left\{\omega^{\prime} \in K_{n}: \mathcal{R}_{f^{n}}\left(\omega^{\prime}\right) \geq \mathcal{R}_{f^{n}}(\omega)\right\}$, then as in the proof of Proposition 5.1, there exists a finite list $\left(a_{i}^{n}\right)_{i \in I_{n}}$ of linear functionals and positive scalars $\left(\alpha_{i}^{n}\right)_{i \in I_{n}}$ such that

$$
R_{n}(\omega)=\left\{x \in E_{n}: a_{i}^{n}(x) \geq \alpha_{i}^{n} \text { for all } i \in I_{n}\right\} .
$$

Moreover, if the family $\left(a_{i}^{n}, \alpha_{i}^{n}\right)_{i \in I_{n}}$ is chosen to be minimal, then $P_{n}(\omega)=\widehat{P}_{n}(\omega) \cap K_{n}$, where

$$
\widehat{P}_{n}(\omega):=\left\{x \in E_{n}: a_{i}^{n}(x)>\alpha_{i}^{n} \text { for all } i \in I_{n}^{\prime}\right\},
$$

and $I_{n}^{\prime}:=\left\{i \in I_{n}: \alpha_{i}^{n}>0\right\}$. Note that $\widehat{P}_{n}(\omega)$ is convex and open in $E_{n}$ and $2 \omega \in \widehat{P}_{n}(\omega)$. Observe in addition that, following Lemma 6.1 of the Appendix, the construction of $\widehat{P}_{n}(\omega)$ is independent of the choice of the minimal family $\left(a_{i}^{n}, \alpha_{i}^{n}\right)_{i \in I_{n}} .{ }^{11}$ We claim that the following property is true.

- The sequence $\left(\widehat{P}_{n}(\omega)\right)_{n \geq 1}$ is increasing, that is, $\widehat{P}_{n}(\omega) \subset \widehat{P}_{n+1}(\omega)$ holds for each $n$.

To see this, let $n \geq 1$. From the definition of $K$ we have $R_{n+1}(\omega) \cap E_{n}=R_{n}(\omega)$. In particular,

$$
R_{n}(\omega)=\left\{x \in E_{n}:\left.a_{i}^{n+1}\right|_{E_{n}}(x) \geq \alpha_{i}^{n+1} \text { for all } i \in I_{n+1}\right\},
$$

where $\left.a_{i}^{n+1}\right|_{E_{n}} \in E_{n}^{*}$ is the restriction of $a_{i}^{n+1}$ to $E_{n}$. Consider a subset $J_{n+1}$ of $I_{n+1}$ such that the family $\left(\left.a_{j}^{n+1}\right|_{E_{n}}, \alpha_{j}^{n+1}\right)_{j \in J_{n+1}}$ is minimal in the definition of $R_{n}(\omega)$. Following the construction of $\widehat{P}_{n}(\omega)$, we have

$$
\widehat{P}_{n}(\omega)=\left\{x \in E_{n}:\left.a_{j}^{n+1}\right|_{E_{n}}(x)>\alpha_{j}^{n+1} \text { for all } j \in J_{n+1}^{\prime}\right\}
$$

where $J_{n+1}^{\prime}=\left\{j \in J_{n+1}: \alpha_{j}^{n+1}>0\right\}$. In particular, $J_{n+1}^{\prime} \subset I_{n+1}^{\prime}$, and $\widehat{P}_{n}(\omega) \subset \widehat{P}_{n+1}(\omega)$.

We are now ready to complete the proof. Start by letting

$$
\widehat{P}(\omega)=\bigcup_{n=1}^{\infty} \widehat{P}_{n}(\omega) .
$$

Clearly, $\widehat{P}(\omega)$ is convex. Since $2 \omega \in \widehat{P}_{1}(\omega)$ it follows that $2 \omega \in \widehat{P}(\omega)$. Moreover, for each $n$ we have $\widehat{P}(\omega) \cap E_{n}=\bigcup_{k=n}^{\infty} \widehat{P}_{k}(\omega) \cap E_{n}$, with $\widehat{P}_{k}(\omega) \cap E_{n}$ open in $E_{n}$. Applying Corollary 2.1 to the

\footnotetext{
${ }^{11}$ Indeed, since $K_{n}$ is generating in $E_{n}$, it follows that $R_{n}(\omega)$ has an interior point in $E_{n}$. Hence, from Lemma 6.1 in the Appendix, the faces of $R_{n}(\omega)$ with dimension $\operatorname{dim} K_{n}-1$ are exactly the convex sets $H_{i} \cap R^{n}(\omega)$ for all $i \in I$, where $H_{i}:=\left\{x \in E_{n}: a_{i}(x)=\alpha_{i}\right\}$.
} 
family $\left(E_{n}\right)_{n \in \mathbb{N}}$ of finite dimensional vector subspaces of $E$, it follows that $\widehat{P}(\omega)$ is $\xi$-open. Now we assert that $\widehat{P}(\omega) \cap K=P(\omega)$. Indeed, $\widehat{P}(\omega) \cap K=\bigcup_{n=1}^{\infty} \widehat{P}(\omega) \cap K_{n}$, and for each $n \geq 1$ we have

$$
\widehat{P}(\omega) \cap K_{n}=\bigcup_{k=n}^{\infty}\left[\widehat{P}_{k}(\omega) \cap K_{k}\right] \cap K_{n}=\bigcup_{k=n}^{\infty} P_{k}(\omega) \cap K_{n}=P_{n}(\omega) .
$$

It follows that $\widehat{P}(\omega) \cap K=\bigcup_{n=1}^{\infty} P_{n}(\omega)=P(\omega)$, and the proof is finished.

\section{Appendix: A note on the faces of a POlyhedral CONVEX SeT}

For notation and terminology not explained in this section, we follow Rockafellar (1970). Let $E$ be a non-trivial finite dimensional vector space and let $A$ be a polyhedral convex set. By definition, there exists a finite family of linear functionals $\left(a_{i}\right)_{i \in I}$ and scalars $\left(\alpha_{i}\right)_{i \in I}$ such that

$$
A=\left\{x \in E: a_{i}(x) \geq \alpha_{i} \text { for all } i \in I\right\} .
$$

The family $\left(a_{i}, \alpha_{i}\right)_{i \in I}$ is said minimal if for each $i \in I$, the set

$$
A^{i}:=\left\{x \in E: a_{j}(x) \geq \alpha_{j} \text { for all } j \neq i\right\}
$$

contains properly $A$.

Again recall that a convex subset $F$ is a face (or an extremal subset) of $A$ if for each line segment $[x, y]$ of $A$ satisfying $(x, y) \cap F \neq \varnothing$ we have $x$ and $y$ belong to $F$.

Lemma 6.1. Let $\mathcal{F}$ be the set of faces of $A$ with dimension $\operatorname{dim} A-1$. If the family $\left(a_{i}, \alpha_{i}\right)_{i \in I}$ is minimal and if $A$ has an interior point then

$$
\mathcal{F}=\left\{H_{i} \cap A: i \in I\right\}
$$

where $H_{i}=\left\{x \in E: a_{i}(x)=\alpha_{i}\right\}$.

Proof. We first prove that for each $i \in I$ the convex set $F_{i}:=H_{i} \cap A$ is a face of $A$ with dimension $\operatorname{dim} A-1$. Let $i \in I$ and let $[x, y] \subset A$ be a line segment such that $(x, y) \cap F_{i} \neq \varnothing$, that is, there exists $\lambda \in(0,1)$ such that $a_{i}(\lambda x+(1-\lambda) y)=\alpha_{i}$. From $a_{i}(x) \geq \alpha_{i}$ and $a_{i}(y) \geq \alpha_{i}$, we get $a_{i}(x)=\alpha_{i}$ and $a_{i}(y)=\alpha_{i}$. We have thus proved that $F_{i}$ is a face of $A$.

Next we shall prove that $\operatorname{dim} F_{i}=\operatorname{dim} A-1$. To this end, let

$$
A^{i}:=\left\{x \in E: a_{j}(x) \geq \alpha_{j} \text { for all } j \neq i\right\}
$$

and observe that $\operatorname{int} A^{i}=\left\{x \in E: a_{j}(x)>\alpha_{j}\right.$ for all $\left.j \neq i\right\}$. Moreover, note that $\left(\operatorname{int} A^{i}\right) \cap H_{i} \subset F_{i}$. Since the family $\left(a_{k}, \alpha_{k}\right)_{k \in I}$ is minimal, we conclude that ${ }^{12}$ (int $\left.A^{i}\right) \cap H_{i} \neq \varnothing$. In particular, we have $\operatorname{dim}\left[\operatorname{int}\left(A^{i}\right) \cap H_{i}\right]=\operatorname{dim} H_{i}=\operatorname{dim} A-1$. But (int $\left.A^{i}\right) \cap H_{i} \subset F_{i} \subset H_{i}$, and hence $\operatorname{dim} F_{i}=\operatorname{dim} A-1$.

Now we shall prove that $\mathcal{F} \subset\left\{F_{i}: i \in I\right\}$. Let $F$ be a face of $A$ with $\operatorname{dimension} \operatorname{dim} A-1$. Then there exists a linear functional $b$ and a scalar $\beta$ such that

$$
F=A \cap\{x \in E: b(x)=\beta\} \text { and } A \subset\{x \in E: b(x) \geq \beta\} .
$$

In particular, we have

$$
\left\{x \in E: a_{i}(x) \geq \alpha_{i} \text { for all } i \in I\right\} \subset\{x \in E: b(x) \geq \beta\} .
$$

\footnotetext{
${ }^{12}$ If (int $\left.A^{i}\right) \cap H_{i}=\varnothing$, then $\operatorname{int} A^{i} \subset\left\{x \in E: a_{i}(x)>\alpha_{i}\right\}$. It follows that int $A^{i} \subset A$, contrary to the fact that $A^{i}$ contains properly $A$.
} 
It follows from Rockafellar (1970) [Theorem 22.3] that there exists a family $\left(\lambda_{i}\right)_{i \in I}$ of non negative scalars $\lambda_{i} \geq 0$ such that

$$
b=\sum_{i \in I} \lambda_{i} a_{i} \text { and } \beta \leq \sum_{i \in I} \lambda_{i} \alpha_{i}
$$

But if $x \in F$, then it follows from $a_{i}(x) \geq \alpha_{i}$ for each $i \in I$ that

$$
\sum_{i \in I} \lambda_{i} \alpha_{i} \leq \sum_{i \in I} \lambda_{i} a_{i}(x)=b(x)=\beta \leq \sum_{i \in I} \lambda_{i} \alpha_{i} .
$$

Consequently, $\beta=\sum_{i \in I} \alpha_{i}$ and hence

$$
F=\left\{x \in E: a_{i}(x) \geq \alpha_{i} \text { for all } i \in I \text { and } \sum_{i \in I} \lambda_{i} a_{i}(x)=\sum_{i \in I} \lambda_{i} \alpha_{i}\right\} .
$$

That is, if $I^{\prime}=\left\{i \in I: \lambda_{i}>0\right\}$, then $F=\bigcap_{i \in I^{\prime}} F_{i}$.

Finally, fix $i \in I^{\prime}$ and note that $F \subset F_{i}$. Since $F$ and $F_{i}$ have the same dimension, it follows that the relative interior of $F$ is contained in the relative interior of $F_{i}$; see Rockafellar (1970) [Corollary 18.1.2]. From this we easily infer that $F=F_{i}$.

\section{REFERENCES}

[1] Aliprantis C. D., D. J. Brown, and O. Burkinshaw (1987): Edgeworth equilibria, Econometrica 55, 11091137.

[2] Aliprantis C. D., D. J. Brown, I. A. Polyrakis, and J. Werner (1998a): Portfolio dominance and optimality in infinite security markets, J. Math. Econom. 30, 347-366.

[3] Aliprantis C. D., D. J. Brown, I. A. Polyrakis, and J. Werner (1998b): Yudin cones and inductive limit topologies, Atti. Sem. Mat. Fis. Univ. Modena 46, 389-412.

[4] Aliprantis C. D., M. Florenzano, and R. Tourky (2002): General equilibrium analysis in ordered topological vector spaces, Working-paper, University Paris 1.

[5] Aliprantis C. D., P. K. Monteiro, and R. Tourky (2003): Non-marketed options, non-existence of equilibria, and non-linear prices, J. Econom. Theory, forthcoming.

[6] Aliprantis C. D., R. Tourky, and N. C. Yannelis (2001): A theory of value: equilibrium analysis beyond vector lattices, J. of Econom. Theory 100, 22-72.

[7] Aubin J.P. (1979): Mathematical Methods of Game and Economic Theory. Amsterdam and New York: NorthHolland.

[8] Bourbaki N. (1966): Espaces Vectoriels Topologiques. 2nd ed., Paris: Herman.

[9] Brown D. J., ANd J. Werner (1995): Arbitrage and existence of equilibrium in infinite asset markets, Rev. Econom. Stud. 62, 101-114.

[10] Debreu G. And H. Scarf (1963): A limit theorem on the core of an economy, Internat. Econom. Rev. 4, $235-246$.

[11] Florenzano M. (1990): Edgeworth equilibria, fuzzy core, and equilibria of a production economy without ordered preferences, J. Math. Anal. Appl. 153, 18-36.

[12] Kelley J. L. and I. Namioka (1963): Linear Topological Spaces. Princeton, NJ: Van Nostrand.

[13] Mas-Colell A. (1986): The price equilibrium existence problem in topological vector lattices, Econometrica 54, 1039-1055.

[14] Rockafellar R. T. (1972): Convex Analysis. 2nd ed., Princeton NJ: Princeton University Press.

[15] Schaefer H. H. (1971): Topological Vector Spaces. New York and Berlin: Springer-Verlag.

[16] Tourky R. (1998): A new approach to the limit theorem on the core of an economy in vector lattices, $J$. Econom. Theory 78, 321-328. 